\title{
Prospective Evaluation of Association Between Negative Emotions and Heart Failure Symptom Severity
}

This article was published in the following Dove Press journal: Psychology Research and Behavior Management

\author{
Kyoung Suk Lee' \\ Terry A Lennie (D) ${ }^{2}$ \\ Debra K Moser ${ }^{2}$ \\ 'Seoul National University, College of \\ Nursing, The Research Institute of \\ Nursing Science, Seoul, South Korea; \\ ${ }^{2}$ University of Kentucky, College of \\ Nursing, Lexington, KY, USA
} by levels of depressive symptoms and anxiety.
Background: Prior studies of symptoms in heart failure (HF) were largely cross-sectional and symptoms were measured using retrospective recall. Because negative emotions influence information processing, retrospective symptom reports by patients with depressive symptoms and anxiety may be biased. The purpose of this study was to determine whether there are differences in patterns of symptom changes, measured prospectively, over 15 days

Methods: HF patients $(\mathrm{N}=52)$ rated daily symptom severity for shortness of breath (SOB), fatigue, sleep disturbance, and edema over 15 days on a 10-point visual analogue scale. Patients were grouped into higher vs lower levels of depressive symptoms and anxiety, respectively, based on median scores of Brief Symptom Inventory subscales. Latent growth curve modeling was used to examine whether patterns of symptom changes over 15 days differed in higher vs lower levels of depressive symptom and anxiety groups.

Results: Those in the higher depressive symptom group had lower levels of baseline symptom severity in $\operatorname{SOB}(\beta:-1.46)$, fatigue $(\beta:-1.71)$, sleep disturbance $(\beta:-1.78)$, and edema $(\beta:-1.97)$ than those in the lower depressive symptom group. However, there were no significant differences in rates of changes in the severity of any of the four symptoms between groups. Anxiety was not associated with baseline severity of symptoms or rates of changes in any of the four symptoms.

Conclusion: Depressive symptoms, but not anxiety, were associated with daily symptom experience. HF patients with higher levels of depressive symptoms may perceive their symptom severity differently than patients with lower levels of depressive symptoms.

Keywords: depressive symptoms, anxiety, heart failure, symptoms
Correspondence: Kyoung Suk Lee Seoul National University, College of Nursing 103 Daehak-ro, Jongno-gu, Seoul 03080, South Korea

Tel +82- 2-740-8829

Fax +82- 2-766-1852

Email kyounglee@snu.ac.kr

\section{Introduction}

Heart failure (HF) is a serious health concern in the United States with high volume and staggering Medicare program expenditure. In the United States, approximately 5.7 million adults have HF, and an additional 3 million will have HF by $2030 .{ }^{1} \mathrm{HF}$ is the most common reason for hospital admissions among Medicare beneficiaries, with $40 \%$ and $65 \%$ of rehospitalization rates at 3 months and 1 year. $^{2}$

Accurate symptom perception is essential for patients with HF to effectively manage their HF, seek care in a timely manner, and prevent repeated hospitalizations. ${ }^{3}$ However, some patients wait up to 7 days or more before seeking medical help. ${ }^{4,5}$ About $40 \%$ of HF patients who had been diagnosed with HF for more than 2 months 
admitted that they were unable to recognize typical symptoms of HF, such as dyspnea, fatigue and orthopnea, as evidence of worsening HF. ${ }^{6}$

The Common-Sense Model of Illness Representations describes how individuals respond to health threats by forming two distinct dimensions of illness representations. ${ }^{7}$ Cognitive illness representations can be shaped based on the information available to the individuals (eg, previous experiences and knowledge). Emotional illness representations capture feelings related to illness. The model suggests that cognitive and emotional illness representations are interrelated and simultaneously influence individuals' responses to health threats. Previous studies have showed that the two dimensions of illness representations are associated with health-related behaviors and outcomes in a variety of groups of patients including patients with $\mathrm{HF}^{8-10}$

Negative emotions, such as depression and anxiety, are barriers to accurate symptom perception because negative emotions affect cognitive processing, such as attention and memory. ${ }^{11-13}$ Patients with higher levels of depressive symptoms and lower levels of anxiety are more likely to have longer delay time from the onset of worsening HF to care-seeking. ${ }^{4,14}$ Depressive symptoms independently predicted the number of physical symptoms that HF patients experienced over 7 days after adjusting for clinical and demographic factors (ie, N-terminal pro-brain natriuretic peptide, age, and race). ${ }^{15}$ These findings suggest that negative emotions influence symptom perception in the HF population.

Howren and Suls found that depressive and anxious moods have a differential effect on retrospective and concurrent symptom reporting. ${ }^{12}$ In their study, depressed mood was related to inflation of retrospective symptom reporting, while anxious mood was related to the inflation of concurrent symptom reporting. ${ }^{12}$ However, prior studies of symptoms in HF in relation to depressive symptoms and anxiety have been mostly cross-sectional and relied on retrospective recall of symptoms (eg, symptom experiences during the past week). ${ }^{15,16}$ This limits our understanding of how the day-to-day symptom experience in HF patients is influenced by depressive symptoms and anxiety.

To address this gap in knowledge, it is important to prospectively investigate the association between $\mathrm{HF}$ symptom assessment and depressive symptoms and anxiety. The purpose of this study was to determine whether there are differences in patterns of symptom severity in four major HF symptoms (ie, shortness of breath [SOB], fatigue, sleep disturbances, and edema) over 15 days between patients with higher and lower levels of depressive symptoms and anxiety, respectively.

\section{Methods}

This study was a prospective, longitudinal study in which we compared patients' symptom ratings for 15 consecutive days by levels of depressive symptoms and anxiety. Patients were recruited from cardiology clinics affiliated with three academic institutions in the United States and Australia. The Institutional Review Boards of all participating institutions (University of Kentucky and Australian Catholic University) approved this study. This study conforms to the principles of the Declaration of Helsinki. Written, signed informed consent was sought and obtained from all participants.

\section{Participants}

Patients $\geq 21$ years of age with a physician-confirmed diagnosis of HF were eligible for this study. Patients were excluded for an acute myocardial infarction in the 3 months prior to the study enrollment, changes in medication regimens in the 3 months prior to the study, a stroke with neurological impairment, life-threatening comorbidities (eg, cancer with active treatment and end-stage renal disease), obvious cognitive impairments, or inability to read or understand English.

\section{Procedures}

Cardiologists and nurses in the clinics consecutively referred patients who met the inclusion/exclusion criteria. Patients were invited to participate in the study and gave signed, written informed consent. After obtaining informed consent, baseline appointments were scheduled. At baseline, patients were asked to complete questionnaires to assess socio-demographic and clinical factors, depressive symptoms, and anxiety. Medical records were reviewed to obtain clinical characteristics, such as left ventricular ejection fraction (LVEF). After patients completed the questionnaires, trained research nurses provided patients with a symptom diary to rate their symptoms for 15 days after returning to home and taught them in detail how to complete the diary (eg, rate symptoms at the same time every day). When patients returned their completed symptom diaries, research nurses reviewed the diary with patients to resolve ambiguities in ratings.

\section{Measures}

\section{Symptom Diary}

Daily symptoms were assessed with the Daily Symptom Scale, ${ }^{17}$ which is a self-report, paper-pencil type of the 
symptom diary. Patients are asked to rate the severity of four symptoms (ie, SOB, fatigue, sleep disturbance, and edema) daily for 15 days. Symptom severity was rated using a 10-point visual analog scale with 0 indicating the absence of symptom and 10 indicating the worst the symptom could be.

\section{Depressive Symptoms and Anxiety}

Depressive symptoms and anxiety were measured using the Brief Symptom Inventory (BSI) subscales for depressive symptoms and anxiety, respectively. ${ }^{18}$ The advantage of using the BSI over other measures (eg, Beck Depression Inventory) is that there are no somatic symptom items, such as sleep disturbances, which may be confounded with HF symptoms and the instrument is short and easy to understand.

There are six items per each subscale of BSI. Patients are asked to rate how much they have been bothered by symptoms of depression or anxiety in the past 7 days on a 5-point scale $(0=$ "not at all" to $4=$ "extremely"). Total scores are obtained by averaging item scores and can range from 0 to 4, with higher scores indicating higher levels of depressive symptoms and anxiety. The validity and reliability of the BSI have been demonstrated. ${ }^{18}$

\section{Sociodemographic and Clinical Data}

Data on following variables were collected via patient interview and medical records review: age, gender, ethnicity, living arrangement, LVEF, New York Heart Association (NYHA) functional classification, comorbidities, and medication regimens. Patients were categorized as having either non-preserved systolic function (LVEF $\leq$ $40 \%$ ) or preserved systolic function (LVEF > 40\%). Trained research nurse carefully conducted in-depth structured patient interviews to determine NYHA classification.

\section{Statistical Analyses}

Medians of baseline scores of the BSI subscale of depressive symptoms (median score $=0.5$ ) and anxiety (median score $=0.5$ ) were used to define higher vs lower levels of depressive symptoms and anxiety because there are only established cut-points for healthy adults. Independent $t$ tests, Mann-Whitney test, chi-square tests, or Fisher's exact tests were used as appropriate to compare characteristics of patients between lower and higher levels of depressive symptoms and anxiety.
Latent growth curve modeling was done with Mplus 7.1. We built separate latent growth curve models by median scores of depressive symptoms (ie, lower vs higher levels of depressive symptoms groups) and anxiety (ie, lower vs higher levels of anxiety groups).

We first built simple latent growth curves for four symptoms (ie, SOB, fatigue, sleep disturbances, and edema) for each depressive symptom groups (ie, higher vs lower), to compare the average trajectory of symptom severity for 15 consecutive days by levels of depressive symptoms. We then incorporated covariates (ie, age, gender, and NYHA class) in the model to determine whether depressive symptom groups are associated with the initial level (ie, intercept) and rate of change (ie, slope) in symptom severity after adjusting for covariates. We followed the same steps described above (latent growth curves modeling without and with covariates) to compare the average trajectory of symptom severity between patients with higher and lower levels of anxiety.

The trajectory of symptom severity is characterized by latent growth factors: an intercept (ie, estimate of baseline level) and linear slope (ie, rate of change over time). Model fit was examined with a standardized root-meansquare residual (SRMR $<0.10$ ), a root-mean-square error of approximation (RMSEA <0.08), a comparative fit index $(\mathrm{CFI}>0.90)$, and Tucker-Lewis Index (TLI >0.90).

Of 3120 symptom diary entries for 52 patients, 23 missing entries were found. Of 23 missing entries, there were no missing entries for two or more consecutive days. We imputed the missing values by averaging before and after the missing values to yield a more complete data set without a loss of power.

\section{Results}

\section{Sample Characteristics}

As shown in Table 1, the mean age of the patients $(n=52)$ was 61 years with a range of 23-87 years. The sample was predominantly Caucasian, male, currently living with someone, and overweight or obese. The majority of the patients had non-preserved systolic function (LVEF $\leq$ $40 \%$ ). All patients were symptomatic (NYHA class IIIV) and more than half of the patients were classified in NYHA III/IV. The most common comorbid conditions were hypertension followed by diabetes.

When comparing sample characteristics between patients with lower and higher levels of depressive symptoms (Table 1), there were no significant differences except for gender 
Table I Sample Characteristics $(\mathrm{N}=52)$

\begin{tabular}{|c|c|c|c|c|c|}
\hline & Total & $\begin{array}{l}\text { Lower Levels of } \\
\text { Depressive Symptoms } \\
(n=26)\end{array}$ & $\begin{array}{l}\text { Higher Levels of } \\
\text { Depressive Symptoms } \\
(n=26)\end{array}$ & $\begin{array}{l}\text { Lower Levels of } \\
\text { Anxiety }(n=27)\end{array}$ & $\begin{array}{l}\text { Higher Levels of } \\
\text { Anxiety }(n=25)\end{array}$ \\
\hline Age, years ${ }^{b}$ & $61 \pm 14$ & $63 \pm 15$ & $60 \pm 13$ & $65 \pm 15$ & $57 \pm 11$ \\
\hline \multicolumn{6}{|l|}{ Gender ${ }^{a}$} \\
\hline Male & $30(57.7)$ & $19(73.1)$ & II (42.3) & $18(66.7)$ & $12(48.0)$ \\
\hline Female & $22(42.3)$ & 7 (26.9) & $15(57.7)$ & $9(33.3)$ & $13(52.0)$ \\
\hline Living with someone & $45(86.5)$ & $23(88.5)$ & $22(84.6)$ & $24(88.9)$ & $21(84.0)$ \\
\hline \multicolumn{6}{|l|}{ Ethnicity } \\
\hline Caucasian & $34(65.4)$ & $17(65.4)$ & $17(65.4)$ & $17(63.0)$ & $17(68.0)$ \\
\hline Others & $18(34.6)$ & $9(34.6)$ & $9(34.6)$ & $10(37.0)$ & $8(32.0)$ \\
\hline Education $(n=5 I)$ & $11 \pm 3$ & $11 \pm 3$ & $12 \pm 3$ & $11 \pm 3$ & $12 \pm 3$ \\
\hline \multicolumn{6}{|l|}{$\begin{array}{l}\text { Body mass index }\left(\mathrm{kg} / \mathrm{m}^{2}\right) \\
(\mathrm{n}=49)\end{array}$} \\
\hline$<25.0$ (normal weight) & $14(28.6)$ & $7(28.0)$ & $7(29.2)$ & $6(24.0)$ & $8(33.3)$ \\
\hline 25.0-29.9 (overweight) & $15(30.6)$ & $8(32.0)$ & $7(29.2)$ & $10(40.0)$ & $5(20.8)$ \\
\hline >30.0 (obese) & $20(40.8)$ & $10(40.0)$ & $10(41.7)$ & $9(36.0)$ & II (45.8) \\
\hline \multicolumn{6}{|l|}{ NYHA Class ${ }^{b}$} \\
\hline II & $24(46.2)$ & $15(57.7)$ & $9(34.6)$ & $16(59.3)$ & $8(32.0)$ \\
\hline $\mathrm{III} / \mathrm{IV}$ & $28(53.8)$ & II (42.3) & $17(65.4)$ & II (40.7) & $17(68.0)$ \\
\hline $\begin{array}{l}\text { Left ventricular ejection } \\
\text { fraction } \leq 40 \%^{\mathrm{b}}(\mathrm{n}=49)\end{array}$ & $40(81.6)$ & 21 (91.3) & $19(73.1)$ & $23(95.8)$ & $17(68.0)$ \\
\hline \multicolumn{6}{|l|}{ Comorbidities } \\
\hline Hypertension & $38(73.1)$ & $16(64.0)$ & $22(84.6)$ & $19(73.1)$ & $19(76.0)$ \\
\hline CVA or stroke & $7(13.5)$ & $2(7.7)$ & $5(19.2)$ & $3(I I . I)$ & $4(16.0)$ \\
\hline Diabetes & $19(36.5)$ & II (42.3) & $8(30.8)$ & $9(33.3)$ & $10(40.0)$ \\
\hline \multicolumn{6}{|l|}{ Medications } \\
\hline ACE I or ARB $(n=5 I)$ & $40(76.9)$ & $20(76.9)$ & $20(80.0)$ & $21(77.8)$ & $19(79.2)$ \\
\hline Beta blockers & $40(76.9)$ & $20(76.9)$ & $20(76.9)$ & $20(74.1)$ & $20(80.0)$ \\
\hline Diuretics & $43(82.7)$ & $21(80.8)$ & $22(84.6)$ & $22(81.5)$ & $21(84.0)$ \\
\hline Digoxin & $21(40.4)$ & $12(46.2)$ & $9(34.6)$ & II (40.7) & $10(40.0)$ \\
\hline Antidepressant & 8 (15.4\%) & $2(7.7 \%)$ & $6(23.1 \%)$ & $3(11.1 \%)$ & $5(20.0 \%)$ \\
\hline Depressive Symptoms ${ }^{\mathrm{a}, \mathrm{b}}$ & $0.96 \pm 0.95$ & $0.19 \pm 0.22$ & $1.74 \pm 0.73$ & $0.4 I \pm 0.64$ & $1.56 \pm 0.87$ \\
\hline Anxiety ${ }^{a, b}$ & $0.73 \pm 0.80$ & $0.24 \pm 0.32$ & $1.22 \pm 0.84$ & $0.17 \pm 0.19$ & $1.33 \pm 0.77$ \\
\hline
\end{tabular}

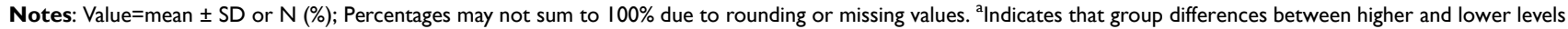
of depressive symptoms; 'Indicates that group differences between higher and lower levels of anxiety.

Abbreviations: NYHA, New York Heart Association; HF, heart failure; CVA, cerebrovascular accident; ACE I, angiotensin converting enzyme inhibitors; ARB, angiotensin II receptor blockers.

and anxiety. Patients with higher levels of depressive symptoms were more likely to be women and have higher levels of anxiety than patients with lower levels of depressive symptoms. Compared with patients with lower levels of anxiety, patients with higher levels of anxiety were more likely to be younger, have preserved systolic function (LVEF $>40 \%$ ), and higher levels of depressive symptoms (Table 1).

\section{Latent Growth Curve Modeling} Depressive Symptom Groups Simple Latent Growth Curve Models

Overall, patients experienced no meaningful rates of changes in SOB, fatigue, sleep disturbance, and edema over 15 days (slopes for the four symptoms $p$-values $>0.05$ ) between patients with lower and higher levels of depressive 


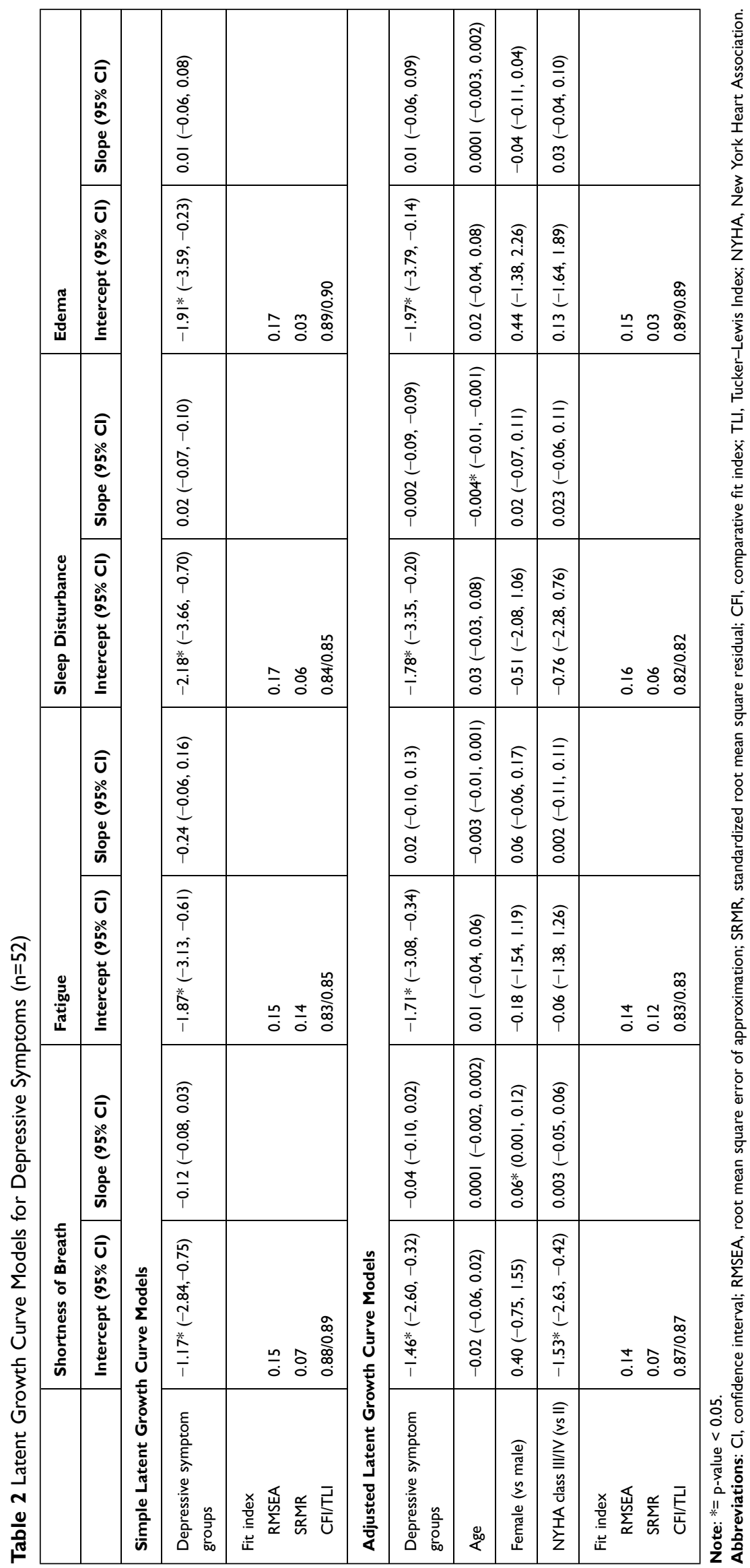


symptoms (Table 2). However, patients with higher levels of depressive symptoms had lower scores of baseline symptom severity (intercept) by 1.17 (95\% confidence interval [CI] = $-2.84,-0.75), 1.87(95 \% \mathrm{CI}=-3.13,-0.61), 2.18(95 \% \mathrm{CI}=$ $-3.66,-0.70)$, and 1.91 points $(95 \% \mathrm{CI}=-3.59,-0.23)$ for $\mathrm{SOB}$, fatigue, sleep disturbance, and edema, respectively, than patients with lower levels of depressive symptoms.

\section{Adjusted Latent Growth Curve Models}

There were no significant differences in rates of changes (slopes) in the severity of any of the four symptoms between the two groups after controlling for age, gender, and NYHA class (Figure 1). However, patients with higher levels of depressive symptoms had lower levels of baseline symptom severity (intercepts) over 15 days in $\operatorname{SOB}(\beta:-1.46,95 \% \mathrm{CI}=$ $-2.60,-0.32)$, fatigue $(\beta:-1.71,95 \% \mathrm{CI}=-3.08,-0.34)$, sleep disturbance $(\beta:-1.78,95 \% \mathrm{CI}=-3.35,-0.20)$, and edema $(\beta$ : $-1.97,95 \% \mathrm{CI}=-3.79,-0.14)$ compared to patients with lower levels of depressive symptoms after adjusting for age, gender and NYHA class (Table 2). This indicates that HF patients with higher levels of depressive symptoms experienced consistently lower symptom severity over 15 days than patients with lower levels of depressive symptoms.

Examining the covariates, women experienced a positive rate of changes in SOB severity ( $\beta$ : $0.06,95 \%$ $\mathrm{CI}=0.001,0.12$ ), indicating a small increase in severity of SOB over 15 days compared to men. Patients in NYHA II reported higher scores of SOB severity at
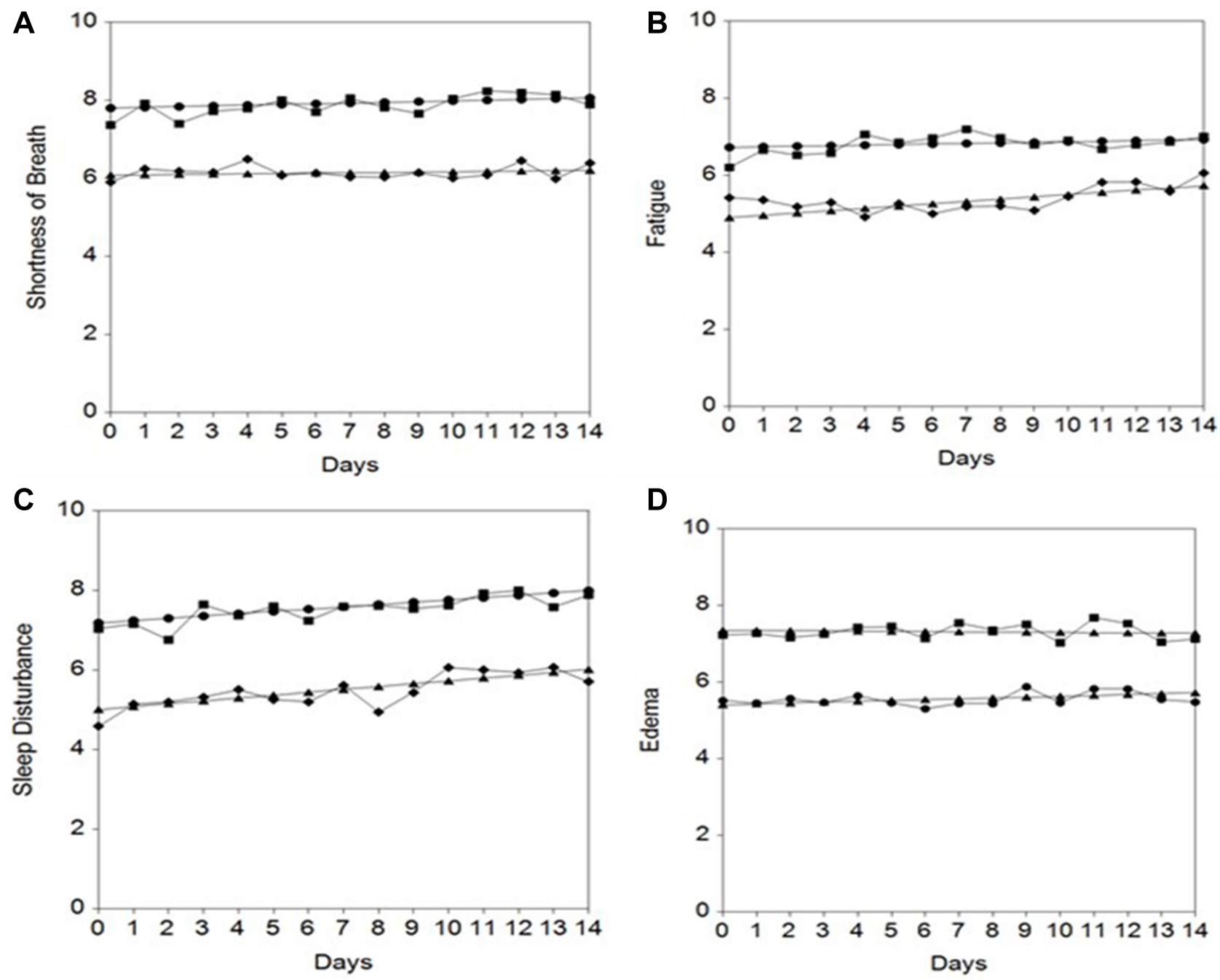

$\mathbf{D}$

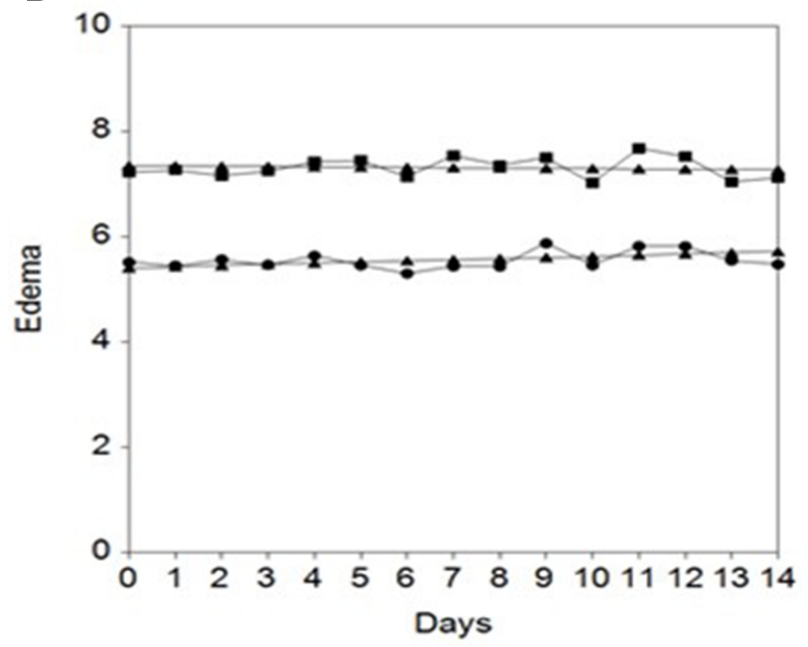

Figure I Trajectory of symptom severity by depressive symptoms $(n=52)$.

Notes: $\boldsymbol{\omega}=$ Lower levels of depressive symptoms (raw). $\bullet=$ Lower levels of depressive symptoms (projected). $\bullet=$ Higher levels of depressive symptoms (raw). $\boldsymbol{\Delta}=$ Higher levels of depressive symptoms (projected). The panels $(\mathbf{A}-\mathbf{D})=$ shortness of breath, fatigue, sleep disturbance, and edema, respectively. $\mathrm{X}$-axis indicates number of days and $y$-axis indicates levels of symptoms. The baseline symptom severity in all four symptoms (shortness of breath, fatigue, sleep disturbance, and edema) was significantly different between patients with higher and lower levels of depressive symptoms. However, there were no significant differences in the rates of changes in any of the four symptoms between the two groups. 


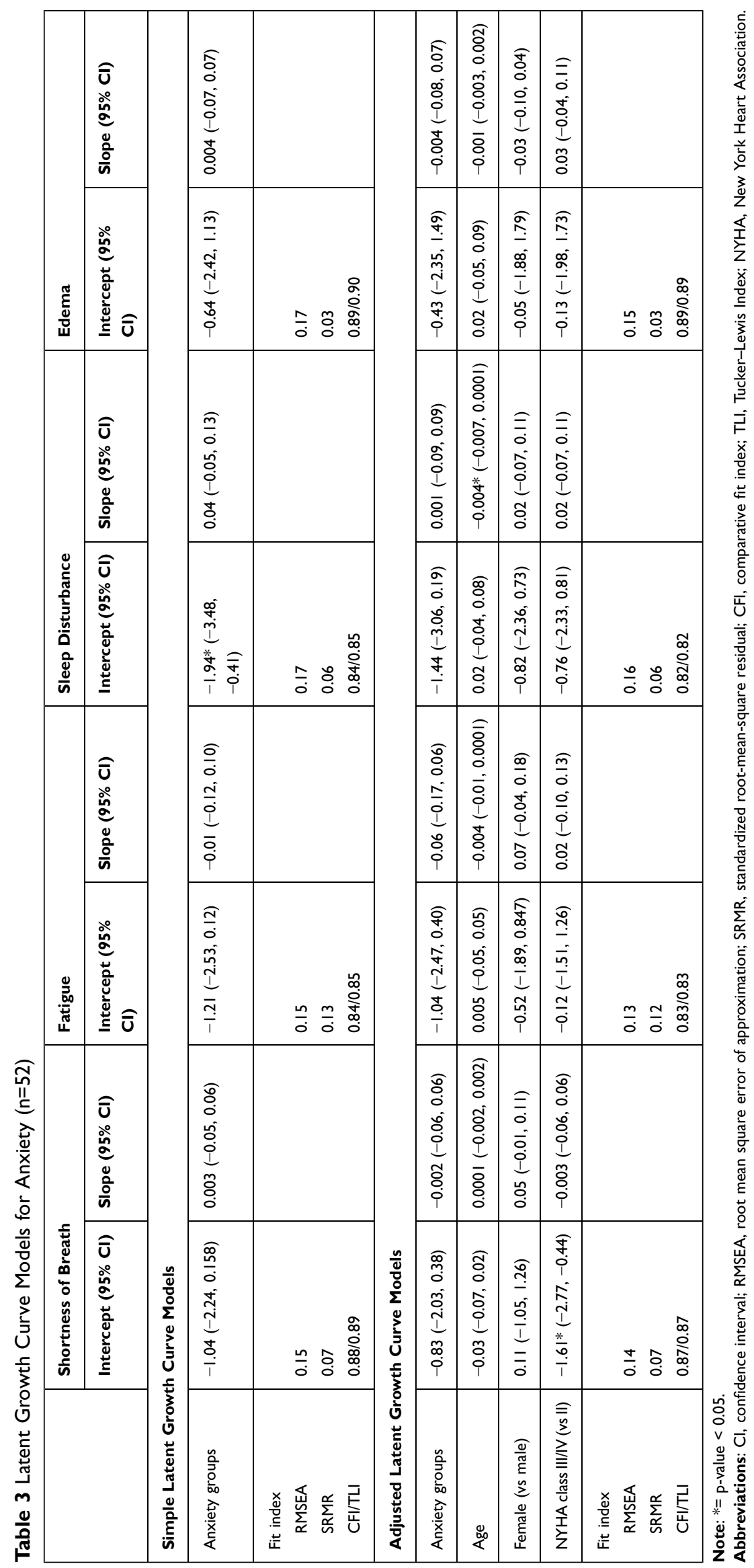




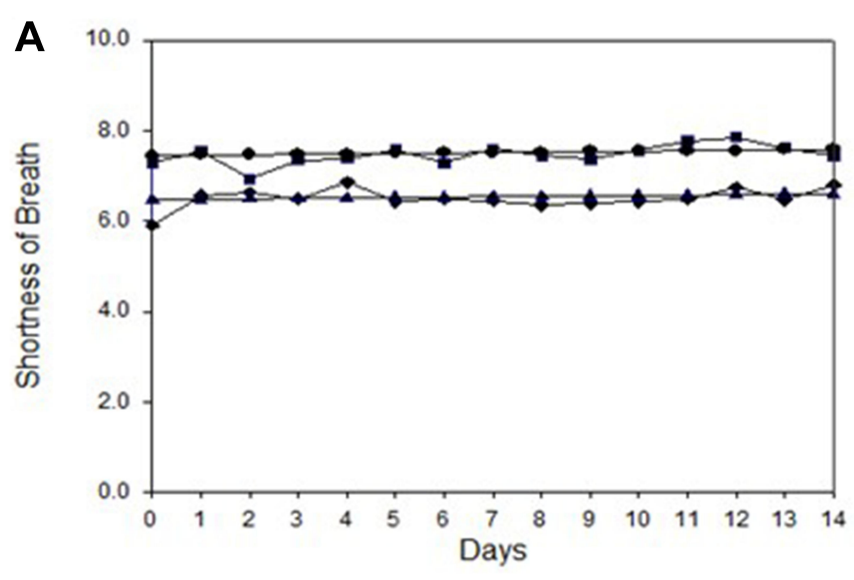

C

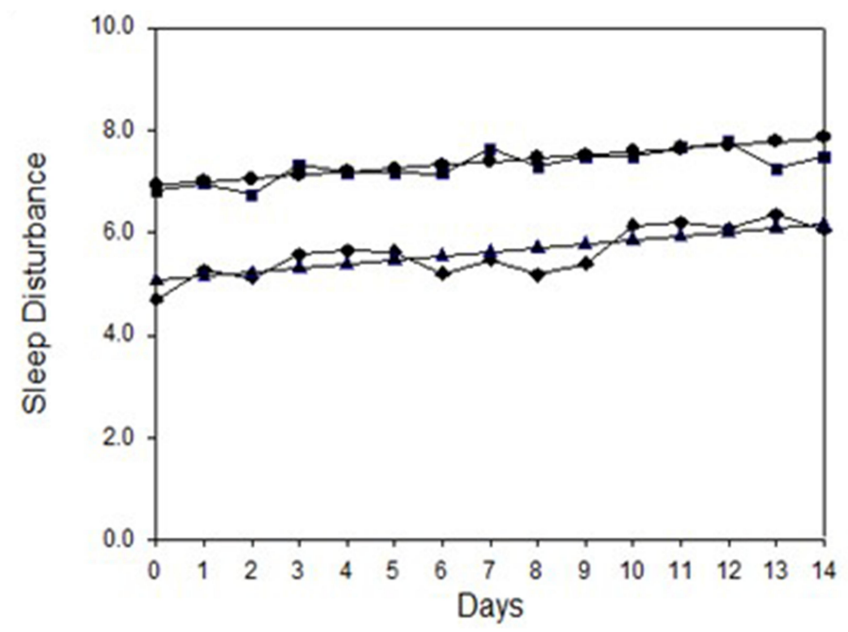

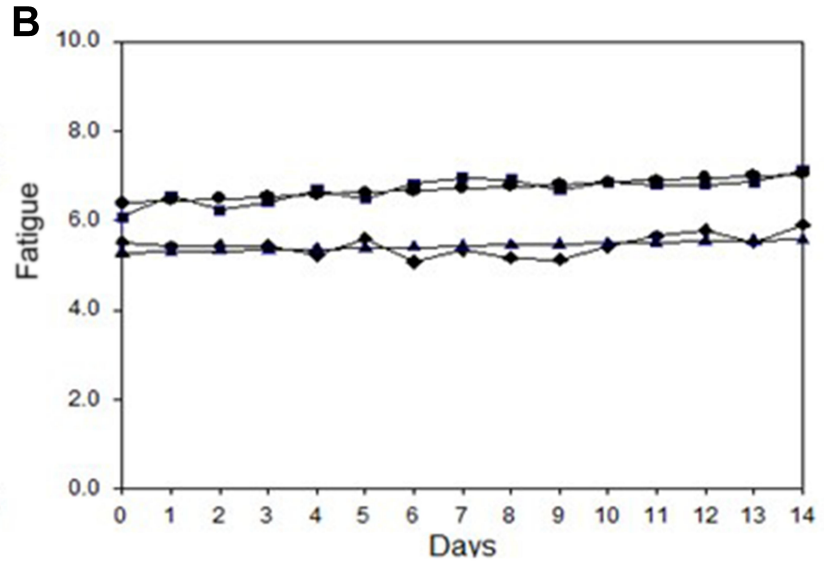

D

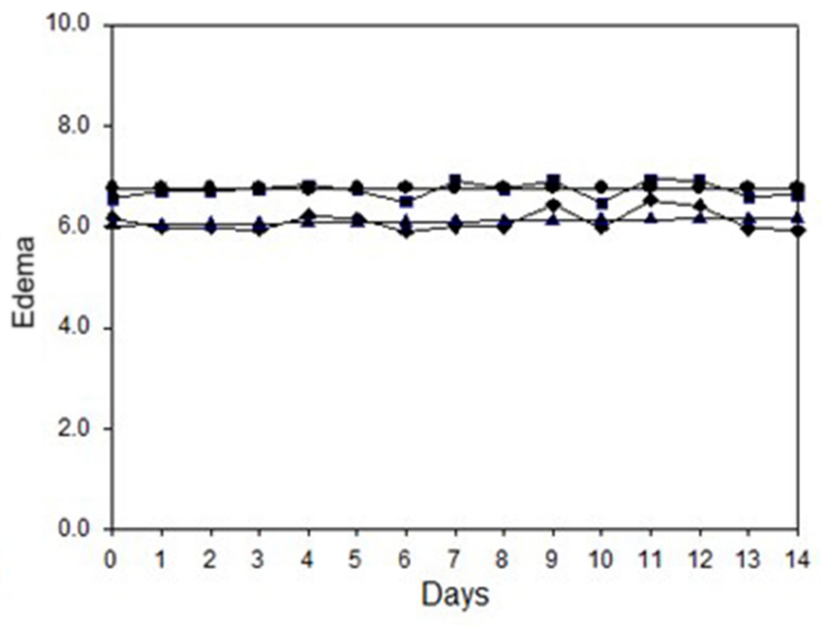

Figure 2 Trajectory of symptom severity by anxiety $(n=52)$.

Notes: $\mathbf{a}=$ Lower levels of anxiety (raw). $\bullet=$ Lower levels of anxiety (projected). $\bullet=$ Higher levels of anxiety (raw). $\boldsymbol{\Delta}=$ Higher levels of anxiety (projected). The panels $(\mathbf{A}-\mathbf{D})=$ shortness of breath, fatigue, sleep disturbance, and edema, respectively. X-axis indicates number of days and y-axis indicates levels of symptoms. The baseline symptom severity in any of the four symptoms (shortness of breath, fatigue, sleep disturbance, and edema) was not significantly different between patients with higher and lower levels of anxiety. There were no significant differences in rates of changes in any of the four symptoms between the two groups.

baseline (intercept) by 1.53 points than patients in NYHA III/IV (95\% CI=-2.63, -0.42). Age was associated with a negative rate of changes in sleep disturbance $(\beta:-0.004$, $95 \% \mathrm{CI}=-0.01,-0.001)$, indicating a very small reduction in severity of sleep disturbance over 15 days with age.

\section{Anxiety Groups}

\section{Simple Latent Growth Curve Models}

There were no significant rates of changes in SOB, fatigue, sleep disturbance, and edema over 15 days (slopes for the four symptoms $p$-values $>0.05$ ) between patients with lower and higher levels of anxiety (Table 3 ). There were no significant differences in baseline symptom severity (intercept) between lower and higher levels of anxiety except for sleep disturbance. Patients with higher levels of anxiety had lower scores of baseline symptom severity (intercept) by 1.94 points $(95 \%$ confidence interval $[\mathrm{CI}]=-3.48,-0.41)$, for sleep disturbance than patients with lower levels of anxiety.

\section{Adjusted Latent Growth Curve Models}

There were no significant differences in rates of changes (slopes) and baseline symptom severity (intercepts) in any of the four symptoms between patients with lower and higher levels of anxiety symptoms, after controlling for age, gender, and NYHA class (Figure 2).

Among covariates, age was associated with a negative rate of changes in sleep disturbance severity $(\beta$ : -0.004 , $95 \% \mathrm{CI}=-0.007,0.0001$ ), indicating a small decrease in severity of sleep disturbance over 15 days as age increases. Patients in NYHA II had higher scores of SOB severity at baseline (intercept) by 1.61 points than patients in NYHA III/IV (95\% CI=-2.77, -0.44). 


\section{Discussion}

This is the first study on the relationship between negative emotions (ie, depressive symptoms and anxiety) and dayto-day HF symptom experiences in patients with HF to be conducted in a prospective manner. In this study, higher levels of depressive symptoms were associated with lower levels of symptom severity, a phenomenon that was stable over time. However, anxiety was not associated with either levels of or changes in symptom severity over time. These results suggest that depressive symptoms and anxiety differentially influence patients' daily symptom experiences. HF patients with higher levels of depressive symptoms appear to perceive their symptom severity differently than patients with lower levels of depressive symptoms. However, anxiety was not associated with symptom experience over 15 days.

Symptoms are subjective sensations indicating a departure from normal function. Symptoms are not merely reflecting functional or structural abnormalities in body systems, but rather the integrated experiences of people in the context of their life situations. ${ }^{19,20}$ Thus, symptoms are experienced in a unique way even among people with the same symptoms and these experiences are influenced by a variety of factors, such as age and emotions. ${ }^{3,21,22}$

Negative emotions, such as depressive symptoms and anxiety, influence information processing processes. ${ }^{11-13}$ Depressed people tend to deeply focus on self, engage less in external environments, be ruminative to stimuli, and recall negative experiences more strongly. ${ }^{22-24}$ These characteristics of depressed people may contribute to an inaccurate symptom perception, particularly symptom reporting from retrospective recalls.

Howren and Suls ${ }^{12}$ conducted an experimental study in which participants were asked two following questions after mood induction: how many physical symptoms were currently experienced and how many physical symptoms had been experienced over 21 days. In their study, participants in a depressed mood induction arm reported a similar number of symptoms compared to those in a neutral mood induction arm. However, participants in a depressed mood induction arm reported a significantly larger number of physical symptoms when they were asked to recall physical symptoms experienced over 21 days. ${ }^{12}$ Similarly, the more depressed patients suffering from low back pain are, the greater inaccuracy in the recall of pain compared to the actual daily diary pain were observed. $^{25}$ These results indicate that depressive symptoms may affect a recall of symptom experiences.

Bekelman and colleagues ${ }^{15}$ demonstrated the positive relationship between depressive symptoms scores and the number of physical symptoms experienced over the past week in patients with HF. This finding reflects the general notion that depressive symptoms may amplify physical symptom complaints in HF. However, in our study HF patients with higher levels of depressive symptoms consistently experienced lower levels of severity of physical symptoms over 15 days. This conflicting finding in our study may be related to the fact that we used a symptom diary to prospectively assess symptoms instead of assessing symptom experiences by relying on recollection over days or weeks. The memory bias in depressive symptoms is consistently found in patients with HF in the present study.

Unlike depression, in which recall bias is a unique characteristic, hypervigilance for threatening information is a distinctive feature of anxiety. ${ }^{24}$ Anxious people have a disproportionate tendency to quickly recognize threatening stimuli compared to neutral stimuli. ${ }^{24,26}$ People in a group where an anxious mood was induced reported a larger number of physical symptoms than people in groups where depressed or neutral moods were induced. ${ }^{12}$ However, in our study anxiety was not related to daily symptom experience. This might be related to the fact that attention bias varies depending on levels of anxiety and degree of intensity of threats. ${ }^{24,27}$ Regarding moderately threatening cues, highly anxious people showed attentional vigilance, while low anxious people showed attentional avoidance. ${ }^{27}$ However, both highly and low anxious people exhibited attentional vigilance to highly threatening cues. ${ }^{27}$ In our study, symptom severities for four physical symptoms ranged from an average of 6-7 out of 10 points, which might not be perceived as highly threatening levels of symptoms by patients. Given that levels of symptom severity in our sample were stable over 15 days, daily similar levels of symptom severity might not be considered as significant cues to patients' HF condition, which contributes to a lack of attention bias on daily symptom experience in our study.

Overall, there were neither positive nor negative rates of changes in symptom severity among HF patients regardless of levels of depressive symptoms and anxiety in our study. This may be related to the fact that we recruited patients from out-patient settings who were relatively stable. As significant changes in weights exhibited 
at least 7 days before hospitalization for $\mathrm{HF}$ decompensation, ${ }^{28}$ it is not expected to observe dramatic fluctuations of symptom severity in stable patients with $\mathrm{HF}$ like the sample in our study.

We found that age was associated with a decrease in the severity of sleep disturbance. However, the rates of changes were so small $(\beta=-0.004$ in both depressive symptoms and anxiety models) that it is unlikely that these rates of changes were clinically significant. The initial scores of symptom severity were not associated with age in our study. However, there are some studies demonstrating age differences in symptom perception. Riegel and colleagues (2010) demonstrated that aging was associated with poor ability to recognize symptoms in patients with HF, which may be explained by changes in the process by which sensory nerve receptors receive and process stimuli that originate inside the body. ${ }^{29}$

Unexpectedly, SOB severity scores of patients in NYHA III/IV were smaller than scores of patients in NYHA II by 1.5 and 1.6 points in depressive symptom and anxiety models, respectively. These counter-intuitive findings may be related to the fact that NYHA functional class was determined by trained research nurses with a structural interview, while SOB severity was rated by patients in their home environment. NYHA functional classes are determined based on the extent to which HF symptoms, such as, but not limited to, SOB, limit physical activity levels. Although NYHA functional class reflects symptom perception to some extent, this is different from patients' perceived SOB severity. A poor agreement between clinician- and HF patient-rated NYHA classes (kappa statistic $=0$. 28) was demonstrated, ${ }^{30,31}$ which may indicate the differences between how functional status was observed by clinicians and perceived by patients themselves.

\section{Implications}

Patients with HF make their decisions to seek care based on their symptom perception. ${ }^{32}$ Our findings suggest that different symptom perception related to depressive symptoms is a reason for worse outcomes in depressed patients in $\mathrm{HF}$. Therefore, it is important for clinicians to recognize the impact of depressive symptoms on symptom perception in HF patients with depressive symptoms to develop strategies to improve the accuracy of their symptom perception. One possible strategy would be a mindfulness-based intervention, which aims at cultivating a non-judgmental, open, and intentional awareness of the present-moment experience (ie, mindful awareness). ${ }^{33}$ From this training, HF patients with depressive symptoms can learn to accurately perceive their symptoms without reflecting rumination.

\section{Limitations}

One limitation of our study is the relatively small sample size with the majority of patients who were white and men. This limits the generalizability of our findings. Data were imputed to minimize a loss of power. However, study results were not altered when using the sample with a complete set of diary data for 15 days. In this study, the daily diary data on symptoms were collected using pencil-and-paper diaries. This type of the diary does not guarantee that patients did complete the diary on time for 15 days although research nurses highlighted the importance of completing the diary at the same time every day. Model fit of the latent growth curve models was not optimal. However, as this study is an exploratory purpose, marginal model fit would be satisfactory for this study. Because the cutoff scores of the BSI for clinically significant depression and anxiety are not established, the median split was used to group patients into lower and higher levels of depressive symptoms and anxiety in this study. In fact, patients classified as higher levels of depressive symptoms and anxiety in this study may not have clinically significant depression and anxiety because the mean scores of the BSI subscales for depressive symptoms and anxiety were 0.96 (SD 0.95) and 0.73 (SD 0.80), respectively. However, the mean scores of the BSI for depressive symptoms and anxiety in previous studies were below $1 .^{34-36}$

\section{Conclusion}

The findings of our study have significant implications in relation to poor outcomes in $\mathrm{HF}$ patients with negative emotions, particularly, depressive symptoms. We found that patient day-to-day symptom experiences were different by levels of depressive symptoms, but not anxiety. Given that care-seeking decisions are made based on symptom perception, inaccurate symptom perception related to depressive symptoms may be a reason for worse outcomes in depressed patients in HF. However, more studies with larger sample sizes are needed to determine why patients with higher levels of depressive symptoms consistently rated their symptom severity lower than patients with lower levels of depressive symptoms. 


\section{Funding}

Source of funding: The National Research Foundation of Korea grant funded by the Korea government (MEST) (2016R1A2B4008495).

\section{Disclosure}

All authors report no conflicts of interest in this work.

\section{References}

1. Roger VL, Go AS, Lloyd-Jones DM, et al. Heart disease and stroke statistics-2012 update. A Report From the American Heart Association. Circulation. 2012;125(1):E2-E220.

2. Greiner MA, Hammill BG, Fonarow GC, et al. Predicting costs among medicare beneficiaries with heart failure. Am J Cardiol. 2012;109(5):705-711. doi:10.1016/j.amjcard.2011.10.031

3. Riegel B, Dickson VV, Cameron J, et al. Symptom recognition in elders with heart failure. J Nurs Scholarsh. 2010;42(1):92-100. doi:10.1111/j.1547-5069.2010.01333.x

4. Jurgens CY, Hoke L, Byrnes J, Riegel B. Why do elders delay responding to heart failure symptoms? Nurs Res. 2009;58(4):274 282. doi:10.1097/NNR.0b013e3181ac1581

5. Goldberg RJ, Goldberg JH, Pruell S, et al. Delays in seeking medical care in hospitalized patients with decompensated heart failure. $\mathrm{Am} \mathrm{J}$ Med. 2008;121(3):212-218. doi:10.1016/j.amjmed.2007.10.032

6. Carlson B, Riegel B, Moser DK. Self-care abilities of patients with heart failure. Heart Lung. 2001;30(5):351-359. doi:10.1067/ mhl.2001.118611

7. Leventhal H, Brissette I, Leventhal EA. The common-sense model of self-regulation of health and illness. In: The Self-Regulation of Health and Illness Behaviour. 2003:42-65.

8. MacInnes J. Relationships between illness representations, treatment beliefs and the performance of self-care in heart failure: a crosssectional survey. Eur J Cardiovasc Nurs. 2013;12(6):536-543. doi: $10.1177 / 1474515112473872$

9. Sawyer AT, Harris SL, Koenig HG. Illness perception and high readmission health outcomes. Health Psychol Open. 2019;6 (1):2055102919844504. doi:10.1177/2055102919844504

10. Richardson EM, Schüz N, Sanderson K, Scott JL, Schüz B. Illness representations, coping, and illness outcomes in people with cancer: a systematic review and meta-analysis. Psycho-oncology. 2017;26 (6):724-737. doi:10.1002/pon.4213

11. Mineka S, Watson D, Clark LA. Comorbidity of anxiety and unipolar mood disorders. Annu Rev Psychol. 1998;49(1):377-412. doi:10.1146/annurev.psych.49.1.377

12. Howren MB, Suls J. The symptom perception hypothesis revised: depression and anxiety play different roles in concurrent and retrospective physical symptom reporting. J Pers Soc Psychol. 2011;100 (1):182-195. doi:10.1037/a0021715

13. Howren MB, Suls J, Martin R. Depressive symptomatology, rather than neuroticism, predicts inflated physical symptom reports in community-residing women. Psychosom Med. 2009;71(9):951-957. doi:10.1097/PSY.0b013e3181b9b2d7

14. Johansson P, Nieuwenhuis M, Lesman-Leegte I, van Veldhuisen DJ, Jaarsma T. Depression and the delay between symptom onset and hospitalization in heart failure patients. Eur J Heart Fail. 2011;13 (2):214-219. doi:10.1093/eurjhf/hfq200

15. Bekelman DB, Havranek EP, Becker DM, et al. Symptoms, depression, and quality of life in patients with heart failure. J Card Fail. 2007;13(8):643-648. doi:10.1016/j.cardfail.2007.05.005

16. Friedman MM, Griffin JA. Relationship of physical symptoms and physical functioning to depression in patients with heart failure. Heart Lung. 2001;30(2):98-104.
17. Webel AR, Frazier SK, Moser DK, Lennie TA. Daily variability in dyspnea, edema and body weight in heart failure patients. Eur $J$ Cardiovasc Nurs. 2007;6(1):60-65. doi:10.1016/j.ejcnurse.2006.04.003

18. Derogatis LR, Melisaratos N. The brief symptom inventory: an introductory report. Psychol Med. 1983;13(3):595-605. doi:10.1017/ S0033291700048017

19. Good B, Good M-J. The Meaning of Symptoms: A Cultural Hermeneutic Model for Clinical Practice. Dordrecht Holland: D. Reidel Publishing Company; 1981.

20. Dodd M, Janson S, Facione N, et al. Advancing the science of symptom management. $J$ Adv Nurs. 2001;33(5):668-676. doi:10.1046/j.1365-2648.2001.01697.x

21. Ekman I, Cleland JG, Andersson B, Swedberg K. Exploring symptoms in chronic heart failure. Eur J Heart Fail. 2005;7(5):699-703. doi:10.1016/j.ejheart.2005.07.003

22. Mathews A, MacLeod C. Cognitive vulnerability to emotional disorders. Annu Rev Clin Psychol. 2005;1(1):167-195. doi:10.1146/ annurev.clinpsy.1.102803.143916

23. Mogg K, Bradbury KE, Bradley BP. Interpretation of ambiguous information in clinical depression. Behav Res Ther. 2006;44 (10):1411-1419. doi:10.1016/j.brat.2005.10.008

24. Mathews A, MacLeod C. Cognitive approaches to emotion and emotional disorders. Ann Rev Psychol. 1994;45(1):25-50. doi:10.1146/annurev.ps.45.020194.000325

25. Lefebvre JC, Keefe FJ. The effect of neuroticism on the recall of persistent low back pain and perceived activity interference. J Pain. 2013;14(9):948-956. doi:10.1016/j.jpain.2013.03.006

26. Williams JM, Mathews A, MacLeod C. The emotional Stroop task and psychopathology. Psychol Bull. 1996;120(1):3-24. doi:10.1037/ 0033-2909.120.1.3

27. Wilson E, MacLeod C. Contrasting two accounts of anxiety-linked attentional bias: selective attention to varying levels of stimulus threat intensity. $J$ Abnorm Psychol. 2003;112(2):212-218. doi:10.1037/0021-843X.112.2.212

28. Chaudhry SI, Wang Y, Concato J, Gill TM, Krumholz HM. Patterns of weight change preceding hospitalization for heart failure. Circulation. 2007;116(14):1549-1554. doi:10.1161/ CIRCULATIONAHA.107.690768

29. Cameron OG. Interoception: the inside story - a model for psychosomatic processes. Psychosom Med. 2001;63(5):697-710. doi:10.1097/ 00006842-200109000-00001

30. Goode KM, Nabb S, Cleland JG, Clark AL. A comparison of patient and physician-rated New York Heart Association class in a community-based heart failure clinic. J Card Fail. 2008;14(5):379-387. doi:10.1016/j.cardfail.2008.01.014

31. Subramanian U, Weiner M, Gradus-Pizlo I, Wu J, Tu W, Murray MD. Patient perception and provider assessment of severity of heart failure as predictors of hospitalization. Heart Lung. 2005;34(2):89-98. doi:10.1016/j.hrtlng.2004.05.002

32. Riegel B, Dickson VV, Faulkner KM. The Situation-Specific Theory of heart failure self-care: revised and updated. J Cardiovasc Nurs. 2016;31(3):226-235. doi:10.1097/JCN.0000000000000244

33. Kabat-Zinn J. Mindfulness-based interventions in context: past, present, and future. Clin Psychol. 2003;10(2):144-156.

34. Khalil AA, Hall LA, Moser DK, Lennie TA, Frazier SK. The psychometric properties of the brief symptom inventory depression and anxiety subscales in patients with heart failure and with or without renal dysfunction. Arch Psychiatr Nurs. 2011;25(6):419-429. doi:10.1016/j.apnu.2010.12.005

35. Hwang B, Moser DK, Dracup K. Knowledge is insufficient for selfcare among heart failure patients with psychological distress. Health Psychol. 2014;33(7):588-596. doi:10.1037/a0033419

36. Lee CS, Gelow JM, Denfeld QE, et al. Physical and psychological symptom profiling and event-free survival in adults with moderate to advanced heart failure. J Cardiovasc Nurs. 2014;29(4):315-323. doi:10.1097/JCN.0b013e318285968a 


\section{Publish your work in this journal}

Psychology Research and Behavior Management is an international, peer-reviewed, open access journal focusing on the science of psychology and its application in behavior management to develop improved outcomes in the clinical, educational, sports and business arenas. Specific topics covered in the journal include: Neuroscience, memory and decision making; Behavior modification and management; Clinical applications; Business and sports performance management; Social and developmental studies; Animal studies. The manuscript management system is completely online and includes a very quick and fair peer-review system, which is all easy to use. Visit http://www. dovepress.com/testimonials.php to read real quotes from published authors. 